\title{
Effect of low-calcium diet and grind diet on bone turnover of ovariectomized female rats
}

\author{
Giselly-Parizoto Costa ${ }^{1}$, Deise-Silva Leite ${ }^{1}$, Renata-Falchete do Prado ${ }^{2}$, Vanessa-Ávila-Sarmento Silveira ${ }^{3}$, \\ Yasmin-Rodarte Carvalho ${ }^{4}$
}

\author{
${ }^{1}$ Undergraduate student, Department of Bioscience and Oral Diagnosis, São José dos Campos School of Dentistry, São Paulo \\ State University - UNESP, São José dos Campos, São Paulo, Brazil \\ ${ }^{2}$ PhD Assistant Professor of Pathology, Department of Phisioterapy, Cruzeiro Superior School - ESC, Cruzeiro, São Paulo, Brazil \\ ${ }^{3} \mathrm{PhD}$ Assistant Professor of Anatomy, Department of Anatomy, Faculty of Pindamonhangaba FAPI, Pindamonhangaba,São \\ Paulo, Brazil \\ ${ }^{4}$ PhD Full Professor of Oral Pathology, Department of Bioscience and Oral Diagnosis, São José dos Campos School of Dentistry, \\ São Paulo State University - UNESP, São José dos Campos, São Paulo, Brazil
}

Correspondence:

Av. Engenheiro Francisco José Longo, 777,

Jardim São Dimas, São José dos Campos,

São Paulo, Brazil CEP 12245000

renatafalchete@hotmail.com Costa GP, Leite DS, do Prado RF, Silveira VA, Carvalho YR. Effect of
low-calcium diet and grind diet on bone turnover of ovariectomized fe-
male rats. Med Oral Patol Oral Cir Bucal. 2011 Jul 1;16 (4):e497-502.
http://www.medicinaoral.com/medoralfree01/v16i4/medoralv16i4p497.pdf

Received: 02/03/2010 Accepted: 27/06/2010

\begin{abstract}
Objective: The variety of methodologies used to investigate ovariectomized female rats shows different results, which makes a clinical application of these results difficult. The purpose of this work was to evaluate the effect of masticatory effort reduction and of low-calcium diet on maxillary bone turnover of ovariectomized female rats. Study Design: Eighty-four female rats were divided into four groups of 21 animals each as follows: SHAM sham-operated; OVZ - ovariectomized and fed a standard commercial diet; LCD - fed a low calcium diet, and GCD - fed a grind commercial diet. The inferior first molars were extracted bilaterally 15 days after the ovariectomy, and the animals were euthanized 3, 5 and 8 weeks after ovaries removal. The maxillae were embedded in methylmetacrilate. The results were submitted to analysis of variance.

Results: The daily mineral apposition rate lowered with time and was not different between SHAM and OVZ groups. The trabecular bone volume of SHAM and OVZ animals was similar and decreased with time. The GCD animals presented the lowest means and the LCD the highest in comparison to the OVZ group.

Conclusion: It was concluded that ovariectomy and a low calcium diet did not cause significant maxillary bone loss in the first molar region, and even in the absence of the antagonist tooth, they did not cause maxillary bone turnover. The grind commercial diet is a good alternative for the study of maxillary bone loss in ovariectomized female rats.
\end{abstract}

Key words: Bone turnover, low calcium diet, ovariectomy. 


\section{Introduction}

Osteoporosis is a systemic disease that leads to bone mass loss and possible maxillary bone compromise (1), which can eventually aggravate periodontal disease (2) and increase dental loss (3). One of the main causes of osteoporosis is the great reduction of estrogen levels in the post-menopause period (4). It is important to understand the pathogenesis of maxillary bone loss for its prevention and also for the establishment of appropriate therapies that minimize bone damage. Therefore, proper methodologies that simulate the process which occurs mainly in the postmenopausal period are necessary. Ovariectomy in adult female rats is a largely used experimental model for the study of the effects of estrogen deficiency on bone since it can be easily performed, it is low cost (5), and shows good results with important clinical applicability in patients with osteoporosis (6-8).

Nevertheless, specifically on maxillary bones, different data has been obtained with the several methodologies applied for the study of bone loss in ovariectomized female rats (8-10), fact that results in a lack of consensus in the literature as for the exact effects of estrogen deficiency on maxillary bone loss (8).

Moriya et al. (11) (1998) verified that the ovariectomy alone does not correspond to a bone loss factor in maxillary bones of female rats. Fact which was probably due to the peculiar masticatory habits of these rodents (12), thus, the administration of a low calcium diet was necessary (8). Alternatively, the ovariectomy experimental model can be associated to a reduction in masticatory effort by means of molar extraction in order to obtain significant bone loss (12).Kiliaridis et al. (13) (1999) observed higher mandibular bone loss when a grind and moist diet was administered (soft diet).

New studies that contribute to improve the model of maxillary bone loss induction related to estrogen deficiency are of great interest to the advance of knowledge related to postmenopausal osteoporosis.

The purpose of the present study was to evaluate the effect of masticatory effort reduction, by means of dental extraction, on maxillary bone loss comparing control and ovariectomized animals. The effect of a low calcium diet in comparison to the effect of a grind diet, in ovariectomized female rats, was also evaluated.

\section{Material and Methods}

\section{Animals}

Eighty-four 90-day-old adult female rats (Rattus norvegicus, variation albinus, Wistar) were used. The animals were supplied by the Animal Breeding Laboratory of the Faculdade de Odontologia de São José dos Campos-UNESP (São José dos Campos Dental School); they were kept in cages at room temperature and fed on a standard commercial diet (Guabi Nutrilabor ${ }^{\circledR}$ ) with calcium content up to $1.2 \%$ and water ad libitum. This study was performed in accordance to the Ethical Principles of Animal Experimentation (COBEA - Brazilian College of Animal Experimentation) and was approved by the local Research Ethics Committee.

The ovariectomy, exodontia and euthanasia were performed under general anesthesia with a solution of $13 \mathrm{mg} / \mathrm{kg}$ of 2-(2.6-xylidino)-5.6-dihydro- $4 \mathrm{H}-1.3$-thiazine hydrochloride (Rompun- Bayer do Brasil) a sedative, analgesic and muscle relaxant; and $33 \mathrm{mg} / \mathrm{kg}$ of ketamine base (Dopalen - Agribands do Brasil Ltda), general anesthetic, intramuscularly.

The groups of 21 rats received the following experimental conditions:

a) SHAM - the group was submitted to sham-operation and was fed a standard commercial diet;

b) OVZ - the group was submitted to bilateral ovariectomy and fed with standard commercial diet;

c) GCD - the group was submitted to bilateral ovariectomy and fed with commercial grind diet;

g) LCD - ovariectomized and fed, after ovariectomy, with low calcium diet $(0.1 \%$ calcium and $0.5 \%$ phosphorus AIN-93M diet - RHOESTER - Indústria e Comércio Ltda, Vargem Grande Paulista, São Paulo, Brazil).

\section{Exodontia}

Fifteen days after the ovariectomy or sham-operation, the lower first molars were extracted. After syndesmotomy and luxation, the teeth were removed and at the end of the procedure the alveoli were sutured with a single stitch. All animals received anti-inflammatory at a dosage of $1 \mathrm{mg} / \mathrm{kg}$ (diclofenac sodium $75 \mathrm{mg}$, Voltaren injectable - Geigy) and $0.1 \mathrm{ml}$ of antibiotic solution (Pentabiótico - Fort Dodge Ltda.).

Tetracycline (Terramicina, Pfizer) was administered, in a dosage of $20 \mathrm{mg} / \mathrm{kg}$, intramuscularly, 24 hours after exodontia and 48 hours before euthanasia. The tetracycline was used as a marker for histomorphometric dynamics with the purpose of verifying the daily mineral apposition rate as previously described (14).

From each group of 21 rats, 7 were euthanized three weeks after the ovariectomy, 7 after 5 weeks and, the remaining 7 eight weeks after abdominal surgery. The animals were euthanized with a lethal injection of anesthetic. The maxillae were removed, dissected, fixed in $10 \%$ formol and sent to resin embedding.

The maxillae were embedded in resin $(85 \%$ methylmethacrylate and $15 \%$ dybutyl phthalate with benzoyl peroxide as a katalizator in kiln at $37^{\circ} \mathrm{C}$ ). Histological cuts were made with its surface perpendicular to the long axis of the molars. Ten microscopic fields were obtained, selected in fluorescence microscope Zeiss Axiophot (2) (Carl Zeiss, Oberköchen, Germany) with original increase of 400X. After that, the images were digitalized with a Sony digital camera (Cybershot model) mounted to the microscope. The histomorphometric analysis of the daily mineral apposition rate was per- 
formed with the aid of the computer program Image-J (National Institutes of Health, for Windows - public domain), by measuring the linear distance between the two fluorescent markers of tetracycline (Fig. 1). The mean daily apposition was obtained for each rat after dividing the time interval of the two injections, expressed in micrometers per day.

Following that, the cuts were dyed with toluidine blue and 5 fields from each cut were photographed in light microscope at original increase of $200 \mathrm{X}$ with the same equipment described above. Image-J was used in order to position a reticulum used to calculate trabecular volume (Vt), which correspond to the volume occupied by bone trabecular, mineralized or not, expressed as a percentage of the volume occupied by bone marrow and trabeculae.

\section{Results}

Histological analysis

A qualitative evaluation of maxillary bone tissue was performed. The area analyzed was the interradicular septum of the left first molar delimited by its five roots (Fig. 2). The osteoclasts were observed in resorption lacunae with low frequency; however, numerous basophilic lines in reversion were observed, indicating remodeling activity. The tissue was cell-rich, composed of many osteocytes and osteoblasts, which were sometimes flat and sometimes columnar, and of areas with osteoid matrix. The organization of periodontal ligament with collagen fibers stretching from the radicular cement to the alveolar bone with irregular contour was observed. The interradicular septum bone showed sometimes concentric lamellar organization and sometimes parallel. The medullary spaces varied in size; however an increase in bone marrow quantity was verified as the cut deepened, that is, became closer to the radicular apex.

The qualitative analysis showed great presence of mastocytes (Fig. 3) distributed by the soft tissues adjacent to the alveolar bone, as well as medullary spaces in the ovariectomized groups in general, differing from the SHAM-operated group.

\section{Daily mineral apposition rate}

The area analyzed was also the interradicular septum of the left first molar delimited by its five roots (Fig. 1). The data was compared statistically according to two approaches. First, the groups SHAM and OVZ were compared considering the following factors as variables: ovariectomy (presence or absence of ovarian hormones) and time of euthanasia in bone turnover. Analysis of Variance test was performed (two-way ANOVA).

In the second approach, the ovariectomized groups that received standard commercial diet, grind commercial diet and low calcium diet were compared by analysis of variance (two-way ANOVA, time of euthanasia and diet). When the analysis of variance showed statistical difference, the Tukey multiple comparison test was performed.

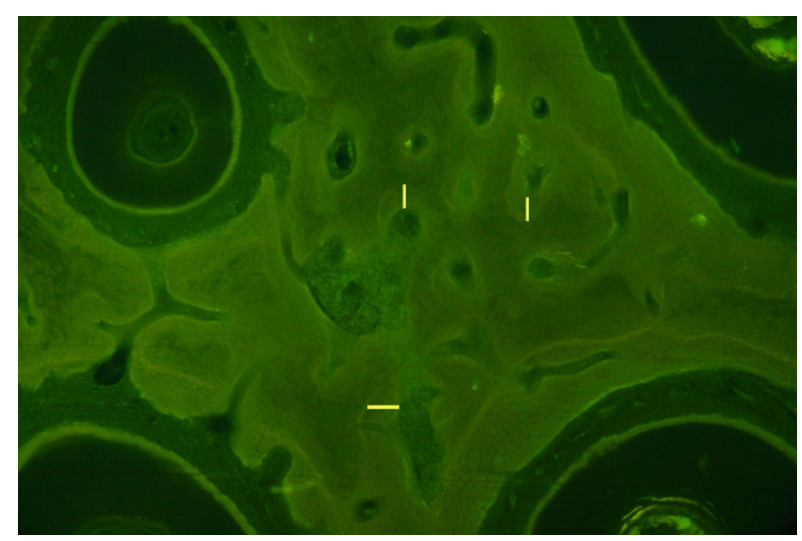

Fig. 1. Photomicroscopy showing 4 roots of first molar and the interradicular bone with fluorescent markers of tetracycline. The yellow lines indicate the local of analysis between two fluorescent marks (original increase 100X, fluorescent microscopy).

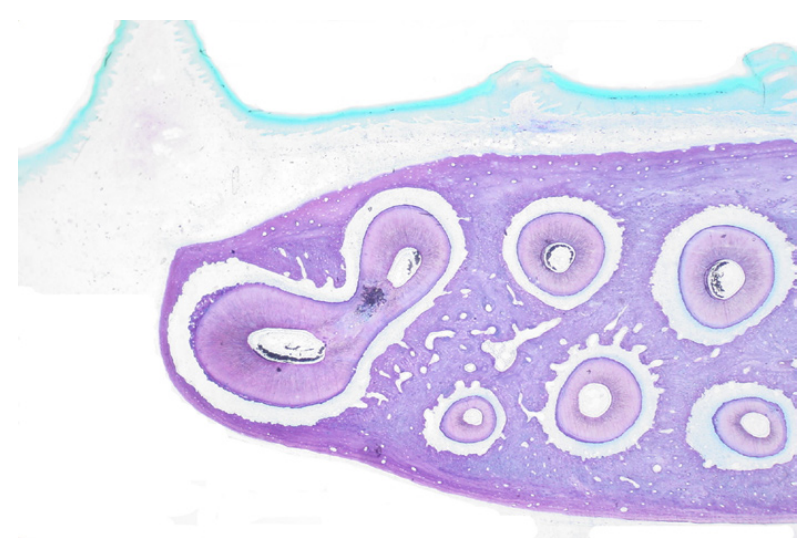

Fig. 2. Panoramic view of the 5 roots of the superior first molars, and 2 mesial roots of the second molar, as well as soft tissues of rugae palatinae (original increase $32 \mathrm{X}$, toluidine blue).

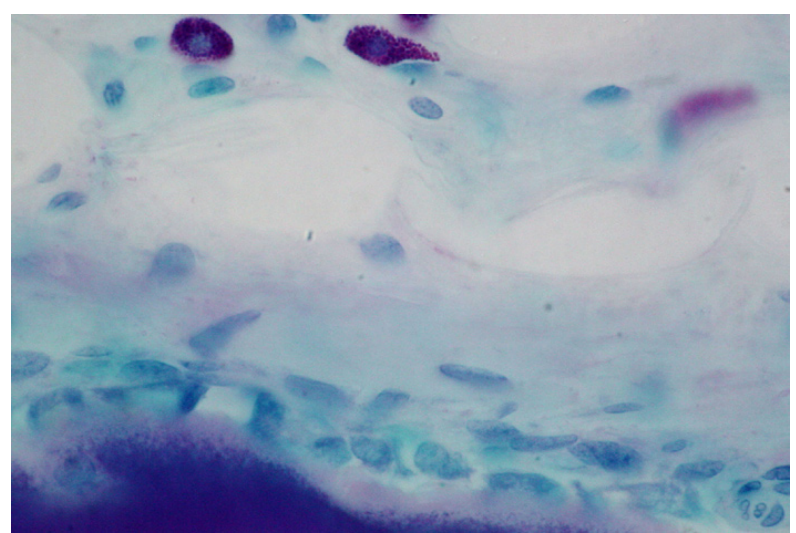

Fig. 3. Mastocytes exhibiting metacromatic granules for toluidine blue and bone surface with osteoid recovered by columnar osteoblasts (original increase $1000 \mathrm{X}$, toluidine blue). 
Table 1 represents the daily mineral apposition rate means in the region near the maxillary first molar.

The ANOVA test showed euthanasia time as significant when the SHAM and OVZ groups were compared $(\mathrm{p}=0.001)$, but not in relation to ovariectomy. The Tukey test, which followed, showed that the daily mineral apposition rate decreased with time, SHAM and OVZ groups were not different.

In relation to the type of diet after ovariectomy, the ANOVA revealed statistical difference; the ovariectomized animals which received grind and low calcium diet were different than the ones that received standard commercial diet (0.001). The factor euthanasia time (0.001), as well as the interaction between time and diet time (0.001) were also significant. According to the Tukey test, GCD and OVZ groups were similar between each other and statistically different than group LCD, being the latter the group with the highest daily mineral apposition rate.

\section{Trabecular volume}

The data were compared statistically according the same two approaches.

Table 2 represent the histomorphometry means with statistical parameters of trabecular bone volume in the region analyzed, in percentage.

The ANOVA test did not show any difference between SHAM and OVZ groups in relation to the presence of hormones $(\mathrm{p}=0.33)$, but they were significant in relation to euthanasia time $(\mathrm{p}=0.003)$. The Tukey test that followed showed that at eight and five weeks, the trabecular volume was statistically lower, regardless of group. The second comparison approach by the ANOVA tests revealed statistical difference among the ovariectomized groups in relation to the diet after surgery. The ovariectomized animals that received grind and low calcium diet were different than the ones fed with standard commercial diet $(p=0.006)$. There was also interaction between euthanasia time and type of diet administered $(\mathrm{p}=0.019)$.

The Tukey test showed that the LCD group obtained lower mean than the OVZ group; however, it was not statistically different. The GCD group showed significant lower trabecular volume means than the OVZ group.

\section{Discussion}

New knowledge that can guide the therapeutic behavior of health professionals who treat women with postmenopausal osteoporosis can contribute for a better quality of life for these patients. The continuous research of the role of estrogen deficiency on the alveolar bone turnover, by several methodologies, that was the aim of this study is, therefore, justified.

Four different experimental groups, with three euthanasia periods each, were used. The first was sham-operated control group (SHAM), the second, ovariectomized

Table 1. Daily mineral apposition rates means \pm standard deviation of groups according to euthanasia time.

\begin{tabular}{|c|c|c|c|c|}
\hline $\begin{array}{c}\text { Euthanasia (weeks } \\
\text { after OVZ) }\end{array}$ & SHAM & OVZ & LCD & GCD \\
\hline 3 & $6.710 \pm 0.79$ & $6.983 \pm 1.059$ & $7.45 \pm 0.69$ & $6.61 \pm 0.56$ \\
\hline 5 & $1.34 \pm 0.12$ & $1.91 \pm 0.26$ & $7.38 \pm 1.13$ & $1.89 \pm 0.29$ \\
\hline 8 & $0.83 \pm 0.07$ & $0.65 \pm 0.09$ & $0.75 \pm 0.10$ & $0.75 \pm 0.12$ \\
\hline
\end{tabular}

SHAM-control; OVZ-ovariectomized standard diet; LCD - ovariectomized low calcium diet; GCD - ovariectomized grind standard diet.

Table 2. Trabecular volume means \pm Standard Deviation of groups according to euthanasia time.

\begin{tabular}{|c|c|c|c|c|}
\hline $\begin{array}{c}\text { Euthanasia (Weeks } \\
\text { after OVZ) }\end{array}$ & SHAM & OVZ & LCD & GCD \\
\hline 3 & $78.88 \pm 7.14$ & $83.86 \pm 9.68$ & $66.62 \pm 13.15$ & $68.15 \pm 10.43$ \\
\hline 5 & $69.65 \pm 7.53$ & $74.67 \pm 6.15$ & $70.25 \pm 9.55$ & $59.55 \pm 8.69$ \\
\hline 8 & $69.21 \pm 11.57$ & $67.23 \pm 9.75$ & $68.46 \pm 4.97$ & $69.59 \pm 9.48$ \\
\hline
\end{tabular}

SHAM-control; OVZ-ovariectomized standard diet; LCD - ovariectomized low calcium diet; GCD ovariectomized grind standard diet. 
control group (OVZ) that received water as a placebo and standard diet. The third group received low calcium diet after ovariectomy (LCD) and the last, grind standard diet (GCD). The purpose of this study was, therefore, to understand the role of calcium deficiency on maxillary bone loss induced by ovariectomy, as well as a possible way to reduce the masticatory effect in order to adequate the experimental model.

Trabecular volume has a direct influence on optical density measured by shades of gray. Therefore, it was expected that for the SHAM group such volume would be higher than for OVZ. Observing the Vt means, it was verified that the SHAM group was slightly superior, but not statistically significant. The difficulty in obtaining osteopenic alterations in gnathic bones of rodents is greatly discussed. Many studies did not find differences between maxillary and mandibular bones of control animals sham-operated and ovariectomized $(8,11)$. This is possibly due to the mastication type of these animals. For this reason, the first mandibular molars of all experimental groups were extracted in order to decrease the masticatory effort and favor bone loss. An alternative would be to preserve the inferior molar in the SHAM group and promote a masticatory effect decrease by extracting this antagonist tooth only in ovariectomized groups, which could lead to different results.

The results for trabecular volume of animals that were fed a low calcium diet were unexpected once the literature reports great bone loss using this methodology $(8,11)$. It was observed that the LCD group mean was lower than the OVZ group; with no statistical significance, however. Uusi-Rasi et al. (15) evaluated women in pre- and postmenopausal phase and observed that the ingestion of calcium after menopause increases femur mineral bone density, the mineral bone density in femurs, especially when these women exercised.

The decreased trabecular volume for the ovariectomized group that received grind standard diet (GCD) when compared to the group that received standard diet (OVZ) presents important information for future studies on maxillary bone turnover with ovariectomy. Masticatory efforts have already been related to bone loss in literature and, as an alternative to this effort reduction, Zaffe et al. (12), Ejiri et al. (16) and Elovic et al. (17) removed molars in association to ovariectomy surgery in order to obtain bone loss in maxillary bones. When the animal suffering in the postoperative period, the time needed for exodontia, expenses with anesthetics, materials, and other factors are considered; the simple administration of grind diet is a more adequate methodology for obtaining maxillary bone loss in osteopenic rats. The studies found in literature about diet thickness did not use this strategy in ovariectomized rats with the purpose of increasing bone loss, in fact, they verified changes in bone development of growing animals (18).
The trabecular volume of rats that received a low calcium diet was slightly superior to the one found in rats fed a grind diet. The low calcium diet was harder when compared to the standard diet, offering higher resistance to animals during mastication, fact that could have caused lower bone loss in the animals that received such diet, in spite of being low calcium. The high cost of this low calcium diet is a disadvantage when compared to the grind standard diet methodology.

In relation to the results obtained for the analysis of daily mineral apposition rate, the ANOVA test did not show differences between SHAM and OVZ groups. There was significant decrease of apposition rate with time, however.

The comparison of the diets showed that the group that received a low calcium diet had the highest daily mineral apposition rate. It is believed that the hormonal deficiency and masticatory effect reduction does not change bone mineral apposition. Despite the extraction of the lower first molar, the harder thickness of the low calcium diet favored bone mineral apposition in the region of the antagonist, probably due to strength dissipation on the other superior molars.

Watanabe et al. (19) performed a cephalometric study in three experimental groups of male growing rats (5 weeks old). The control group was fed a standard diet for six weeks; the moderate calcium privation group was fed a low calcium diet for 4 weeks and a standard diet in the last 2 experimental weeks; and the last group was fed a low calcium diet for the whole experimental period of 6 weeks. The cephalometric analysis showed that the superoinferiror growth (vertical) was retarded and decreased in the low calcium groups, while the posteroanterior growth (horizontal) was accelerated in these groups. This study was performed in Japan and the authors did not mention the thickness difference of the two used diets as described in the present work. The authors suggest that such results may be due to the dental contour and masticatory habits of the animals.

In the present study, the histological analysis showed abundant presence of mastocytes. The mastocytes proliferative disturbances cover a number of lesions whose symptoms are related to inflammatory mediator liberation which include redness, rash and hives. Among these symptoms, it can be cited matocytic hyperplasia or reactive mastocytosis, which can be observed in the bone marrow of women with postmenopausal osteoporosis (20). These cells are possibly important in the bone turnover process resulting from estrogen deficiency.

\section{Conclusion}

Based on the experimental conditions of these work, it can be concluded that the estrogen deficiency associated or not to calcium deficiency did not cause maxillary bone loss in the region of the first molar, even in the absence 
of the antagonist tooth. The use of grind commercial diet to reduce the masticatory effort of ovariectomized rodents resulted in significant trabecular volume reduction and is shown as a possible alternative to the study of maxillary bone loss in ovariectomized rats.

\section{References}

References with links to Crossref - DOI

1. Binte Anwar R, Tanaka M, Kohno S, Ikegame M, Watanabe N, Nowazesh Ali M, et al. Relationship between porotic changes in alveolar bone and spinal osteoporosis. J Dent Res. 2007;86:52-7.

2. Shen EC, Gau CH, Hsieh YD, Chang CY, Fu E. Periodontal status in post-menopausal osteoporosis: a preliminary clinical study in Taiwanese women. J Chin Med Assoc. 2004;67:389-93.

3. Yoshihara A, Seida Y, Hanada N, Nakashima K, Miyazaki H. The relationship between bone mineral density and the number of remaining teeth in community-dwelling older adults. J Oral Rehabil. 2005;32:735-40.

4. Manolagas SC, Jilka RL. Bone marrow, cytokines, and bone remodeling. Emerging insights into the pathophysiology of osteoporosis. N Engl J Med. 1995;33:305-11.

5. Wuttke W, Jarry H, Becker T, Schultens A, Christoffel V, Gorkow $\mathrm{C}$, et al. Phytoestrogens: endocrine disrupters or replacement for hormone replacement therapy? Maturitas. 2003;44 Suppl 1:S9-20.

6. Picherit C, Bennetau-Pelissero C, Chanteranne B, Lebecque P, Davicco MJ, Barlet JP, et al. Soybean isoflavones dose-dependently reduce bone turnover but do not reverse established osteopenia in adult ovariectomized rats. J Nutr. 2001;131:723-8.

7. Omi N, Ezawa I. The effect of ovariectomy on bone metabolism in rats. Bone. 1995; 17:163S-168S.

8. Teófilo JM, Azevedo AC, Petenusci SO, Mazaro R, Lamano-Carvalho TL. Comparison between two experimental protocols to promote osteoporosis in the maxilla and proximal tibia of female rats. Pesqui Odontol Bras. 2003;17:302-6.

9. Ishihara A, Sasaki T, Debari K, Furuya R, Kawawa T, Ramamurthy NS, et al. Effects of ovariectomy on bone morphology in maxillae of mature rats. J Electron Microsc (Tokyo). 1999;48:465-9.

10. Elovic RP, Hipp JA, Hayes WC. Ovariectomy decreases the bone area fraction of the rat mandible. Calcif Tissue Int. 1995;56:305-10.

11. Moriya Y, Ito K, Murai S. Effects of experimental osteoporosis on alveolar bone loss in rats. J Oral Sci. 1998;40:171-5.

12. Zaffe D, Paganelli C, Cocchi D. Induction and pharmacological treatment of oral osteopenia in rats. Minerva Stomatol. 1999;48:4562.

13. Kiliaridis S, Thilander B, Kjellberg H, Topouzelis N, Zafiriadis A. Effect of low masticatory function on condylar growth: a morphometric study in the rat. Am J Orthod Dentofacial Orthop. 1999;116:121-5.

14. Parfitt AM, Drezner MK, Glorieux FH, Kanis JA, Malluche H, Meunier PJ, et al. Bone histomorphometry: standardization of nomenclature, symbols, and units. Report of the ASBMR Histomorphometry Nomenclature Committee. J Bone Miner Res. 1987;2:595610 .

15. Uusi-Rasi K, Sievänen H, Pasanen M, Beck TJ, Kannus P. Influence of calcium intake and physical activity on proximal femur bone mass and structure among pre-and postmenopausal women. A 10year prospective study. Calcif Tissue Int. 2008;82:171-81.

16. Ejiri S, Toyooka E, Tanaka M, Anwar RB, Kohno S. Histological and histomorphometrical changes in rat alveolar bone following antagonistic tooth extraction and/or ovariectomy. Arch Oral Biol. 2006;51:941-50.

17. Elovic RP, Hipp JA, Hayes WC. Maxillary molar extraction causes increased bone loss in the mandible of ovariectomized rats. J Bone Miner Res. 1995;10:1087-93.

18. Yamamoto S. The effects of food consistency on maxillary growth in rats. Eur J Orthod. 1996;18:601-15.

19. Watanabe K, Imamura H, Uchikanbori S, Fujita Y, Maki K. Effects of restricted calcium intake on bone and maxillofacial growth. Angle Orthod. 2008;78:445-52.
20. Donker ML, Bakker NA, Jaspers WJ, Verhage AH. Two patients with osteoporosis: initial presentation of systemic mastocytosis. J Bone Miner Metab. 2008;26:199-202.

\section{Acknowledgments}

The authors would like to thank The State of São Paulo Research Foundation (FAPESP) for the financial support provided as a scientific initiation scholarship, process n. 07/53807-8 and 07/53806-1. 